RESEARCH HIGHLIGHT

\title{
How did a novel X-linked gene become essential for male
} determination?

\author{
Scott William Roy ${ }^{1 凶}$ \\ (c) CEMCS, CAS 2021
}

Cell Research (2022) 32:7-8; https://doi.org/10.1038/s41422-021-00577-8

\begin{abstract}
A new study reports a necessary role in male sex determination for the X-linked gene SDX. Remarkably, scrutiny reveals that SDX evolved long after the male-determining gene $S R Y$, raising the question of how a new gene could become essential for a crucial, ancient process.
\end{abstract}

While $S R Y$ is generally said to be the sex-determining gene in mammals, the full story is more complicated. First, there exist people who develop female genitalia but who have XY chromosomes with an intact SRY gene (female genital development in $X Y$ people is known as Swyer syndrome). ${ }^{1}$ Second, in some rodent species, there exist so-called feminizing $X$ chromosomes, in which common $X$-linked mutations (in unknown genes) lead $X Y$ individuals to develop as females. ${ }^{2,3}$ Finally, $X Y$ females have also been described in various species, without explanation. ${ }^{3}$ Writing in Cell Research, Zhan and coauthors report the first known X-linked gene that is necessary for male sex determination in mammals. ${ }^{4}$ SDX (also known as PWWPB3 and MUM1L1) is necessary for male development, as knockout of the gene leads to remarkably complete male-to-female sex reversal in some $X Y$ individuals, and reduced spermatogenesis in non-reversed individuals. The authors show that $S D X$ presence in XY mice affects expression of both $S R Y$ and its major downstream target, SOX9, but not upstream regulators of $S R Y$; the simplest explanation for this pattern would be that $S D X$ directly upregulates $S R Y$. $S D X$ is an immediate candidate for mutations leading to Swyer syndrome in humans as well as for mutations that may be involved in the evolution of feminizing $X$ chromosomes in rodents.

Further scrutiny reveals that $S D X$ is in fact a highly atypical $X$ chromosomal gene. The full scenario is depicted in Fig. 1. The mammalian $X$ and $Y$ chromosomes evolved from an ancestral autosomal pair in the ancestor of marsupials and placental mammals, with additional sequence added later. ${ }^{5}$ Conserved synteny indicates that the vast majority of modern X-linked genes were present on these ancestral autosomes, suggesting that for most genes X-linkage represents simple lack of movement. ${ }^{6}$ However, SDX is only found in placental mammals, suggesting that it evolved more recently, as a retroposed copy of the ancestral MUM1 gene found on chromosome $19^{5}$ (Fig. 1). Such a history is rare, with only a few dozen X-linked retroposed mammalian genes out of a total of hundreds of X-linked genes ${ }^{7}$. Notably, retroposed genes show a strong tendency towards testis expression, consistent with SDX's function in early male determination.

This relatively recent evolutionary history also indicates that SDX is a highly atypical sex determination gene. Genes involved in sex determination in animals are famously ancient. ${ }^{8}$ For instance, homologs of DMRT1, which has independently evolved sex determination roles in diverse vertebrates, are involved in sex determination in flies and worms. Similarly, homologs of SOX3 and $A M H$ evolved sex-determining roles independently multiple times. By contrast, SDX is a new gene and appears to have newly acquired a role in sex determination: no sex-determining role is known for any homolog, and knockout of its parent gene MUM1 yields no sex determination phenotype (Mengcheng Luo, personal communication). While little is known about SDX, MUM1 and related PWWP domain-containing proteins tend to function in chromatin remodeling by interacting with other core chromatinrelated proteins including DNA methylatransferases. ${ }^{9}$ This modality suggests that SDX could affect various loci, in which case SDX could promote male development not by specifically upregulating $S R Y$, but by more generally promoting gene expression at an early stage of embryogenesis in which $S R Y$ expression is crucial for male development.

$S D X ' s$ relatively recent origin implies $S D X$-independent $S R Y$-based male sex determination in ancestral mammals and in modern marsupials, raising the question of how SDX could have secondarily became necessary for such a crucial function in placental mammals. One possibility invokes tradeoffs experienced by ancient sex determination regulators. Like most vertebrates, mammalian SRYdriven sex determination involves highly cross-regulated genes specific to early male (Sox9, DMRT1, AMH, SF1) or female (WNT4, RSPO1, Fox/2) development. These autosomal sex determination genes must balance robust expression in one sex with robust silencing in the other sex. This dual requirement may be in tension if for instance mutational changes that ensure robust expression of a male-specific gene in males lead to incomplete silencing in females and vice versa. Under such dynamics, genes may evolve to 'split the difference', tolerating low levels of intersex phenotypes in both sexes.

The exception to these tradeoffs is $S R Y$. Because it is male specific, an increase in $S R Y$ expression at crucial stages (in particular, early embryogenesis) entails no costs in females. Ever more robust $S R Y$ expression driven by SDX could change the tradeoffs experienced by ancient regulators. Increased SDX-driven SRY expression could decrease the marginal benefits of male-specific gene expression in males without affecting the costs in females, thus tipping the balance towards less aggressive expression of male-specific genes. These dynamics would be expected to produce a ratchet: increased SRY promotion by SDX would lead to decreased promotion of malespecific gene expression by other factors, driving further selection for

\footnotetext{
${ }^{1}$ Department of Biology, San Francisco State University, San Francisco, CA, USA. ${ }^{凶}$ email: scottwroy@gmail.com
} 
The discovery of the first known X-linked gene necessary for male sex determination in mammals highlights the complexity of sex determination systems as well as the evolutionary histories and selective dynamics that shape these systems. It will be interesting to learn more about SDX's mechanism of regulation and its regulatory roles beyond sex determination. Probing the involvement of SDX's orthologs and paralogs in sex determination and related gene regulatory networks will also be crucial to understanding the evolutionary steps by which SDX evolved roles in male sex determination. These findings highlight how much there is to be learned about core sex determination networks in even the best-studied species.

\section{REFERENCES}

Fig. 1 Schematics of sex chromosome evolution in mammals. Modern mammalian $X$ chromosomes date to the evolution of SRY from SOX3 and initial $X / Y$ differentiation (blue/red), in the shared marsupial/placental ancestor. Subsequently, SDX arose by a retroposition of the autosomal gene MUM1 onto the $X$ chromosome. Additional events included acquisition of additional sequence from an autosome (dark gray) and ongoing $X / Y$ differentiation and $Y$ degradation. Times from timetree.org.

increased SDX-driven $S R Y$ expression. Given such dynamics, SDX could become ever more important for male sex determination over time, to the point that SDX becomes necessary for expression of male-specific genes.

The new results also place a spotlight on which mutations are likely to underlie feminizing rodent $X$ chromosomes, whose existence could play outsized roles in the evolution of novel sex determination systems, sex chromosome drive, speciation and social structures. ${ }^{2}$ Interestingly, feminizing $X$ chromosomes often exhibit autosomal fusions, ${ }^{10}$ which could suggest that ancestrally autosomal factors are responsible, for unknown reasons. Notably, because the fused autosomal region remains present in two copies per cell, feminizing mutations in autosome-derived genes would seemingly need to be dominant, for instance through dominant-negative regulatory phenotypes.

1. Hawkins, J. R. Human Mutat. 2, 347-350 (1993).

2. Burt, A. \& Trivers, R. Harvard University Press, Cambridge, MA, (2009).

3. Bull, J. J. \& Bulmer, M. G. Heredity 47, 347-365 (1981).

4. Zhan, J., Cui, P., Yu, Z., Qu, W. and Luo, M. Cell Res. https://doi.org/10.1038/s41422021-00539-0 (2021).

5. Kumar, S., Stecher, G., Suleski, M. \& Hedges, S. B. Mol. Biol. Evol. 34, 1812-1819 (2017).

6. Ross, M. T. et al. Nature 434, 325-337 (2005).

7. Emerson, J. J., Kaessmann, H., Betrán, E. \& Long, M. Science 303, 537-540 (2004).

8. Graves, J. A. M. \& Peichel, C. L. Genome biology 11, 205 (2010).

9. Fagerberg, L. et al. Mol. Cell. Proteom. 13, 397-406 (2014).

10. Baudat, F., de Massy, B. \& Veyrunes, F. Chromosoma 128, 397-411 (2019).

\section{COMPETING INTERESTS}

The authors declare no competing interests.

\section{ADDITIONAL INFORMATION}

Correspondence and requests for materials should be addressed to Scott William Roy.

Reprints and permission information is available at http://www.nature.com/ reprints 